\title{
Pesticides side-effects
}

Integrated Pest Management (IPM) evolved for more than 50 years as an alternative to chemical control eco-friendly approach providing practical, economically viable and societal acceptable solutions for pest control. The integration of biological control with pesticides use is a desirable, though a difficult to accomplish goal of modern IPM. Only during the last decades, the development of the modern groups of 'Reduced-Risk' and 'Biorational' insecticides with reduced side-effects to biological control agents (BCAs) or limited persistence under field conditions has created new options for combining chemical and biological control in greenhouse crops. Herein, the two groups are briefly presented together with data on the classification of main representative chemicals based on their toxicity to BCAs.

\section{Pesticides side-effects}

Integrated Pest Management (IPM) protocols have been developed and implemented for more than 50 years for several greenhouse crops. In these IPM programs, chemical, cultural and biological control options have been integrated to achieve the desired control of key pests. However, during the early phase of IPM implementation in greenhouse crops, pest control programs mainly relied on broad spectrum pesticides, thus leaving limited options for the conservation of biological control agents (BCAs) naturally occurring or introduced in the crop.

The use of efficient pesticides to control key pest populations, but still selective or less toxic to natural enemies has been recognized as the key point in successful integration of chemical with biological control. Therefore, information on the side effects of pesticides on BCAs has been collected using several different protocols used to evaluate their toxic lethal and/or sublethal effects under laboratory, semi-field and/or field conditions. The most widely accepted toxicological protocols for several different groups of BCAs have been developed by the International Organization for Biological Control (IOBC) - WPRS Working Group 'Pesticides and beneficial organisms'.

In principle, insecticides of well-documented low toxicity or short persistence under field conditions should be considered as compatible with BCAs conservation. However, selectivity of different insecticides may vary considerably among BCA species. This is the case for example for closely related species belonging even to the same family (e.g. phytoseiid predatory mites), for which considerable variation has been recorded in their susceptibility to certain selective pesticides. Detailed knowledge of the toxicological profile of the available pesticides relative to the BCAs released in greenhouse crops is essential for the successful integration of chemical and biological control.
Currently, two pesticides groups termed 'biorational' and 'Reduced-Risk Insecticides' (RRIs), respectively monopolize researchers' attention working on the implementation of IPM programs in greenhouses. While belonging to two distinct groups distinguished mainly by their origin (biologicallyderived or not, respectively) confusion still exists in worldwide literature, mainly with respect to the inclusion of RRIs to biorational insecticides.

\section{Biorational insecticides}

Naturally derived products, such as plant extracts (e.g. azadirachtin), microbial pesticides, insecticides derived from soil microorganisms (e.g. spinosyns: spinosad, spinetoram; avermectins: abamectin, emamectin benzoate), and plantderived oils all belong to the biorational pesticides group. Lack of toxicity to BCAs and/or short persistence under field conditions are the main characteristics of this group, therefore justifying their compatibility with conservation biological control (Table 1).

\section{Reduced-risk insecticides}

A far more diverse group of modern synthetic insecticides, RRIs, are of high specificity to herbivorous pests and of low toxicity to mammals, as well as of high selectivity to BCAs.

Neonicotinids (e.g. imidacloprid thiamethoxam acetamiprid, thiacloprid), diamides (e.g. flubendiamide, chlorantraniliprole), Insect Growth Regulators (IGRs) (ecdysone agonists: tebufenozide methoxyfenozide; juvenile hormone mimics: fenoxycarb pyriproxyfen; chitin synthesis inhibitors: buprofezin, lufenuron, novaluron, diflubenzuron, teflubenzuron, flufenoxuron; moulting disruptors: Cyromazine), as well as certain novel groups of molecules like pyridine (pymetrozine) and oxadiazines (indoxacarb) are the main representatives of RRIs. Due to their specific traits favouring selectivity to BCAs, they currently form the most promising chemical control option within the context of IPM programs. 
Table 1. Toxicity of selected insecticides to the most important BCA families currently used in greenhouse vegetable and ornamental crops for biological control of insect and mite pests. Toxicity class 1 pesticides are the safest to be applied in combination with BCAs.

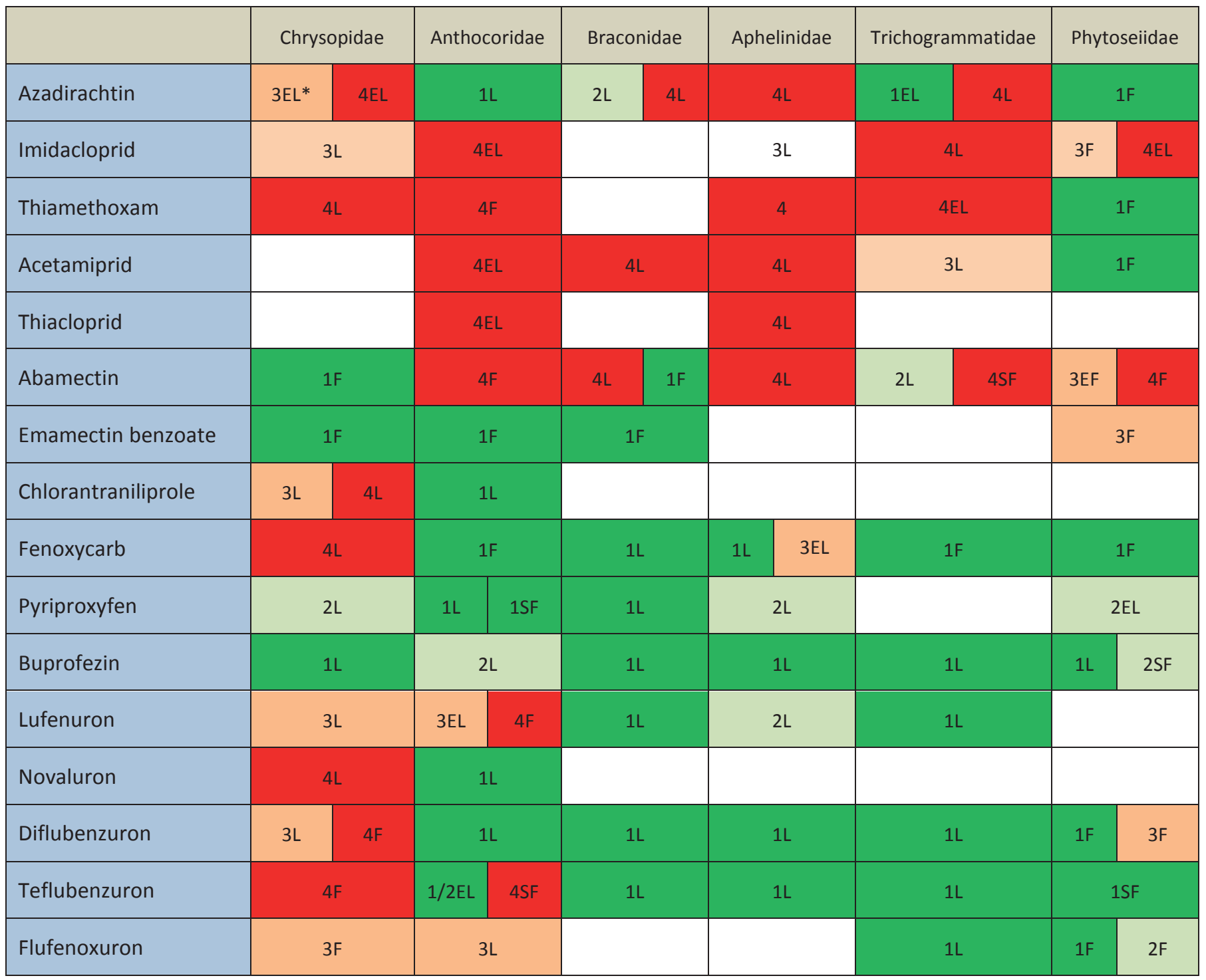

*L= Initial Toxicity Lab; EL=Extended Lab; SF= Semi field; F= Field test

IOBC toxicity classes:

L: 1 (Harmless) $\mathrm{E}<30 \%, 2$ (Slightly harmful) $\mathrm{E}=30-79 \%, 3$ (Moderately harmful) $\mathrm{E}=80-99 \%, 4$ (Harmful) $\mathrm{E}>99 \%$

EL / SF / F: 1 (Harmless) E<25\%, 2 (Slightly harmful) $E=25-50 \%, 3$ (Moderately harmful) $E=50-75 \%, 4$ (Harmful) $E>75 \%$

\section{Information sources on pesticide side- effects on BCAs}

A user searchable database on selectivity of pesticides to BCAs is available at the IOBC webpage (IOBC Pesticide Side-Effects Database).

References: Döker I., M. L. Pappas, K. Samaras, A. Triantafyllou, C. Kazak and G. D. Broufas. 2014. Compatibility of reduced-risk insecticides with the non-target predatory mite Iphiseius degenerans (Acari: Phytoseiidae), Pest Management Science, DOI: 10.1002/ps.3921.

Messelink, G. J., J. Bennison, O. Alomar, B. L. Ingegno, L. Tavella, L. Shipp, E. Palevsky, and F. L. Wäckers. 2014. Approaches to conserving natural enemy populations in greenhouse crops: current methods and future prospects. BioControl 59: 377-393.
Acknowledgement: This work was supported by COST Action FA1105 "Towards a sustainable and productive EU organic greenhouse horticulture".

DOI: http://dx.doi.org/10.18174/373602

Authors: G.D. Broufas

Affiliation: Democritus University of Thrace, Greece gbroufas@agro.duth.gr

January 2016 